Volume 141, Number 12, December 2013, Pages 4191-4197

S 0002-9939(2013)11730-2

Article electronically published on August 22, 2013

\title{
RESULTS ON WITT KERNELS OF QUADRATIC FORMS FOR MULTI-QUADRATIC EXTENSIONS
}

\author{
ROBERTO ARAVIRE AND AHMED LAGHRIBI
}

(Communicated by Matthew A. Papanikolas)

\begin{abstract}
In this paper we compute the Witt kernel of quadratic forms for the composition of a purely inseparable multi-quadratic extension with a separable quadratic extension. We also include the case of a multi-quadratic purely inseparable extension by completing the proof given before by the second author for such an extension.
\end{abstract}

Throughout this paper $F$ denotes a field of characteristic 2. Let $W_{q}(F)$ (resp. $W(F)$ ) be the Witt group of nonsingular quadratic forms over $F$ (resp. the Witt ring of regular bilinear forms over $F$ ). The group $W_{q}(F)$ is endowed with a $W(F)$ module structure as follows: To a bilinear form $(V, B)$ and a nonsingular quadratic form $(W, \varphi)$ with the polar form $B_{\varphi}$, we associate a nonsingular quadratic form $B \otimes \varphi$ defined on $V \otimes_{F} W$ by: $B \otimes \varphi(v \otimes w)=B(v, v) \varphi(w)$ for $(v, w) \in V \times W$, whose polar form is $B \otimes B_{\varphi}[\mathrm{B}$.

For an integer $n \geq 1$, let $I^{n} F$ denote the $n$-th power of the fundamental ideal $I F$ of $W(F)\left(\right.$ set $\left.I^{0} F=W(F)\right)$, and let $I^{n} W_{q}(F)$ denote the group $I^{n} F \otimes W_{q}(F)$.

We denote by $\left\langle a_{1}, \cdots, a_{n}\right\rangle_{b}$ the $n$-dimensional diagonal bilinear form $\sum_{i=1}^{n} a_{i} x_{i} y_{i}$ for $a_{1}, \ldots, a_{n} \in F^{*}:=F \backslash\{0\}$. The 2-dimensional quadratic form $a x^{2}+x y+b y^{2}$ will be denoted by $[a, b]$.

A given field extension $K / F$ induces natural homomorphisms $i_{K}: W_{q}(F) \longrightarrow$ $W_{q}(K)$ and $j_{K}: W(F) \longrightarrow W(K)$. Let $W_{q}(K / F)$ and $W(K / F)$ denote the kernels of $i_{K}$ and $j_{K}$, respectively.

An important problem in the algebraic theory of quadratic (bilinear) forms is the computation of $W_{q}(K / F)$ and $W(K / F)$. These kernels are known for some field extensions. For example, for $K / F$ purely transcendental, these kernels are trivial. If $K / F$ is separable, then $W(K / F)$ is trivial since an anisotropic $F$-bilinear form remains anisotropic over a separable extension of $F$. For $K / F$ inseparable, the kernel $W(K / F)$ was computed by Hoffmann in $[\underline{\mathrm{H}}]$. For $K$ the function field of a hypersurface (including algebraic simple extensions), the kernel $W(K / F)$ was computed by Dolphin and Hoffmann in [DH. Before this result the second author computed $W(K / F)$ when $K$ is the function field of a quadric [L1]. Still for $K$ the function field of a quadric, some partial results on $W_{q}(K / F)$ are given in [1]. Also, for $\varphi$ a bilinear Pfister form, the kernel $I^{m}(F(\varphi) / F)$ was computed by Baeza and the first author in $[\mathrm{AB}]$.

Received by the editors April 2, 2011 and, in revised form, February 13, 2012.

2010 Mathematics Subject Classification. Primary 11E04, 11E81.

Key words and phrases. Quadratic forms, Witt kernels, differential forms.

The first author was supported by Fondecyt \#1090006 and Proyecto Anillos PBCT ACT056. 
In this paper we are interested in the kernel $W_{q}(K / F)$ when $K / F$ is a multiquadratic extension. As the characteristic is 2 , there are two kinds of quadratic extensions: the inseparable quadratic extension $F[t] /\left(t^{2}-a\right)$ with $a \in F \backslash F^{2}$, which we denote by $F(\sqrt{a})$, and the separable quadratic extension $F[t] /\left(t^{2}+t+b\right)$ with $b \notin \wp(F)$, which we denote by $F\left(\wp^{-1}(b)\right)$ (here $\wp: F \longrightarrow F$ is the Artin-Schreier operator given by: $\left.x \mapsto x^{2}-x\right)$.

In the case of a purely inseparable multi-quadratic extension $K / F$, the kernel $W_{q}(K / F)$ was computed in [L2], but it turns out that the proof contains a mistake. So our first aim in this paper is to give a complete proof of the following theorem (see comments after Proposition 1):

Theorem 1 ([L2, Th. 1]). For any scalars $a_{1}, \cdots, a_{n} \in F$, we have the following:

$$
W_{q}\left(F\left(\sqrt{a_{1}}, \cdots, \sqrt{a_{n}}\right) / F\right)=\sum_{i=1}^{n}\left\langle 1, a_{i}\right\rangle_{b} \otimes W_{q}(F) .
$$

After that, based on Theorem 1, we will compute the kernel of the composition of a purely inseparable extension with a quadratic separable extension:

Theorem 2. For any scalars $a_{1}, \ldots, a_{n}, b \in F$, we have the following:

$$
W_{q}\left(F\left(\wp^{-1}(b), \sqrt{a_{1}}, \cdots, \sqrt{a_{n}}\right) / F\right)=W(F) \otimes[1, b]+\sum_{i=1}^{n}\left\langle 1, a_{i}\right\rangle_{b} \otimes W_{q}(F) .
$$

Previously, Theorem 1 was proved by Mammone and Moresi [MM, Th. 2(i)] for $n=2$, and by Baeza [B] Lem. 4.3, page 182] for $n=1$. Theorem[2] is due to Ahmad for $n=1$ [Ah]. In [B, Cor. 4.16, page 128], Baeza gave the computation in the case of quadratic and bi-quadratic separable extensions: $W_{q}\left(F\left(\wp^{-1}(a), \wp^{-1}(b)\right) / F\right)=$ $W(F) \otimes[1, a]+W(F) \otimes[1, b]$. But this computation does not extend to tri-quadratic separable extensions as was proved by Mammone and Moresi [MM, Prop. 1].

Our proofs are reduced to computations on differential forms. This is allowed by the well known result of Kato [K], which gives the relation between nonsingular quadratic forms and differential forms (see below).

Let us recall some facts about differential forms. For any integer $n \geq 1$, we denote by $\Omega_{F}^{n}=\bigwedge^{n} \Omega_{F}^{1}$ the space of $n$-differential forms $\left(\Omega_{F}^{0}=F\right)$, where $\Omega_{F}^{1}$ is the $F$-vector space generated by the symbols $d x, x \in F$, subject to the relations $d(x+y)=d x+d y$ and $d(x y)=x d y+y d x$ for $x, y \in F$. In particular, there is a $F^{2}$-linear map $F \longrightarrow \Omega_{F}^{1}$, given by: $x \mapsto d x$. This map extends to the differential operator $d: \Omega_{F}^{n} \longrightarrow \Omega_{F}^{n+1}$ as follows: $d\left(x d x_{1} \wedge \cdots \wedge d x_{n}\right)=d x \wedge d x_{1} \wedge \cdots \wedge d x_{n}$. The usual Artin-Schreier operator $\wp: F \longrightarrow F, \wp(a)=a^{2}-a$, extends to the general Artin-Schreier map

$$
\wp: \Omega_{F}^{n} \longrightarrow \Omega_{F}^{n} / d \Omega_{F}^{n-1}
$$

given on generators by

$$
\wp\left(x \frac{d x_{1}}{x_{1}} \wedge \cdots \wedge \frac{d x_{n}}{x_{n}}\right)=\left(x^{2}-x\right) \frac{d x_{1}}{x_{1}} \wedge \cdots \wedge \frac{d x_{n}}{x_{n}}+d \Omega_{F}^{n-1} .
$$

Let $\nu_{F}(n)=\operatorname{Ker}(\wp)$ and $H_{2}^{n+1}(F)=\operatorname{Coker}(\wp)$. By a result of Kato [K], there exists an isomorphism

$$
e^{n}: \overline{I^{n}} W_{q}(F) \longrightarrow H_{2}^{n+1}(F)
$$


given on generators as follows:

$$
e^{n}\left(\left\langle\left\langle a_{1}, \cdots, a_{n}\right\rangle\right\rangle_{b} \otimes[1, c]+I^{n+1} W_{q}(F)\right)=c \frac{d a_{1}}{a_{1}} \wedge \cdots \wedge \frac{d a_{n}}{a_{n}}+\wp\left(\Omega_{F}^{n}\right)+d \Omega_{F}^{n-1},
$$

where $\overline{I^{n}} W_{q}(F)$ denotes the quotient $I^{n} W_{q}(F) / I^{n+1} W_{q}(F)$ and $\left\langle\left\langle a_{1}, \cdots, a_{n}\right\rangle\right\rangle_{b}$ denotes the $n$-fold bilinear Pfister form $\left\langle 1, a_{1}\right\rangle_{b} \otimes \cdots \otimes\left\langle 1, a_{n}\right\rangle_{b}$. In [K], Kato also established a relation between bilinear forms and differentials forms, but we will not need this fact here.

Let $\left\{a_{i} \mid i \in I\right\}$ be a 2-basis of $F$; i.e., the set $\left\{\prod_{i \in I} a_{i}^{\epsilon_{i}} \mid \epsilon_{i} \in\{0,1\}\right.$, and $\epsilon_{i}=$ 0 for almost all $i \in I\}$ is an $F^{2}$-basis of $F$. We fix some ordering on $I$ and consider the set $\sum_{n}:=\{\sigma:\{1, \cdots, n\} \longrightarrow I \mid \sigma(i)<\sigma(j)$ whenever $i<j\}$. This set $\sum_{n}$ is equipped with the lexicographic ordering. Hence, the set $\left\{\frac{d a_{\sigma}}{a_{\sigma}} \mid \sigma \in \sum_{n}\right\}$ is an $F$-basis of $\Omega_{F}^{n}$, where $\frac{d a_{\sigma}}{a_{\sigma}}=\frac{d a_{\sigma(1)}}{a_{\sigma(1)}} \wedge \cdots \wedge \frac{d a_{\sigma(n)}}{a_{\sigma(n)}}$ for any $\sigma \in \sum_{n}$. For $\alpha \in \sum_{n}$, let $\Omega_{F, \alpha}^{n}\left(\right.$ resp. $\left.\Omega_{F,<\alpha}^{n}\right)$ be the subspace of $\Omega_{F}^{n}$ generated by $\frac{d a_{\sigma}}{a_{\sigma}}$ with $\sigma \leq \alpha$ (resp. the subspace of $\Omega_{F}^{n}$ generated by $\frac{d a_{\sigma}}{a_{\sigma}}$ with $\sigma<\alpha$ ). Hence, we get a filtration of $\Omega_{F}^{n}$ given by $\Omega_{F, \alpha}^{n}, \alpha \in \sum_{n}$ (and $\Omega_{F,<\alpha}^{n}, \alpha \in \sum_{n}$ ). For any $i \in I$, let $F_{i}$ be the subfield $F^{2}\left(a_{j} \mid j \leq i\right)$.

A crucial result that we will use in our proofs is due to Kato. We will refer to it as Kato's lemma:

Proposition $1([\overline{\mathrm{K}}])$. Let $\alpha \in \sum_{n}$ and $y \in F$ be such that $\wp\left(y \frac{d a_{\alpha}}{a_{\alpha}}\right) \in \Omega_{F,<\alpha}^{n}+$ $d \Omega_{F}^{n-1}$. Then, there exist $v \in \Omega_{F,<\alpha}^{n}$ and $c_{i} \in F_{\alpha(i)}^{*}, 1 \leq i \leq n$, such that

$$
y \frac{d a_{\alpha}}{a_{\alpha}}=\frac{d c_{1}}{c_{1}} \wedge \cdots \wedge \frac{d c_{n}}{c_{n}}+v .
$$

Clearly, this proposition implies that any element $\eta \in \nu_{F}(n)$ can be written as $\eta=\sum_{\beta \leq \alpha} u_{\beta} \frac{d l_{\beta}}{l_{\beta}}$ for some $\alpha \in \sum_{n}, l_{\beta(i)} \in F_{\beta(i)}^{*}, u_{\beta}=0,1$, and $\frac{d l_{\beta}}{l_{\beta}}=\frac{d l_{\beta(1)}}{l_{\beta(1)}} \wedge \cdots \wedge$ $\frac{d l_{\beta(n)}}{l_{\beta(n)}}$.

The following proposition is incorporated in [L2, Prop. 1], and it is the basis of Theorem 1. When trying to prove Theorem 2 we noticed that some details in the proof of [L2, Prop. 1] should be corrected (see the explanations in the following footnote). This is the reason why we reproduce the proof here.

Proposition 2. For any integer $n \geq 1$ and scalars $\alpha_{1}, \cdots, \alpha_{m} \in F$, we have

$$
\operatorname{Ker}\left(H_{2}^{n+1}(F) \longrightarrow H_{2}^{n+1}\left(F\left(\sqrt{\alpha_{1}}, \cdots, \sqrt{\alpha_{m}}\right)\right)\right)=\sum_{i=1}^{m} \overline{d \alpha_{i} \wedge \Omega_{F}^{n-1}(F)} .
$$

Proof. Let $L=F\left(\sqrt{\alpha_{1}}, \cdots, \sqrt{\alpha_{m}}\right)$ and $M=F\left(\sqrt{\alpha_{1}}\right)$. Without loss of generality, we may suppose that $[L: F]=2^{m}$. So we can choose a 2-basis $\mathcal{B}=$ $\left\{c_{1}, \cdots, c_{m}, c_{m+1}, \cdots\right\}$ of $F$ such that $c_{i}=\alpha_{i}$ for $i=1, \cdots, m$.

We proceed by induction on $m$. The case $m=1$ is done in [AB, Lem. 2.18]. Suppose that the proposition is true for any field $F^{\prime}$ of characteristic 2 and any purely inseparable multi-quadratic extension $L^{\prime} / F^{\prime}$ such that $\left[L^{\prime}: F^{\prime}\right]<2^{m}$.

Since $[L: M]=2^{m-1}$, we get by the induction hypothesis that $\operatorname{Ker}\left(H_{2}^{n+1}(M) \longrightarrow\right.$ $\left.H_{2}^{n+1}(L)\right)=\sum_{i=2}^{m} \overline{d \alpha_{i} \wedge \Omega_{M}^{n-1}}$. 
Let $\omega \in \Omega_{F}^{n}$ be such that $\bar{\omega} \in \operatorname{Ker}\left(H_{2}^{n+1}(F) \longrightarrow H_{2}^{n+1}(L)\right)$. Then, over $M$, we have $\bar{\omega} \in \operatorname{Ker}\left(H_{2}^{n+1}(M) \longrightarrow H_{2}^{n+1}(L)\right)$. Hence, $\bar{\omega} \in \sum_{i=2}^{m} \overline{d \alpha_{i} \wedge \Omega_{M}^{n-1}}$. This means that there exist $u \in \Omega_{M}^{n}$ and $v \in \Omega_{M}^{n-1}$ such that

$$
w=\sum_{i=2}^{m} \frac{d \alpha_{i}}{\alpha_{i}} \wedge \sigma_{i}+\wp(u)+d(v),
$$

for suitable $\sigma_{2}, \cdots, \sigma_{m} \in \Omega_{M}^{n-1}$. For the sequel we use a descent argument 11 in order to reduce to the case where $\sigma_{1}, \cdots, \sigma_{m}, u, v$ are defined over $F$.

The set $\mathcal{B}^{\prime}:=\left\{c_{2}, \cdots, c_{m}, c_{m+1}, \cdots, \sqrt{\alpha_{1}}\right\}$ is a 2-basis of $M$. We may suppose that $\sqrt{\alpha_{1}}$ is the last element in $\mathcal{B}^{\prime}$. Let $\delta$ be the maximal multi-index in (11).

Suppose that $\delta(1)>m$. Then we get

$$
w_{\delta} \frac{d c_{\delta}}{c_{\delta}}=\wp\left(u_{\delta} \frac{d c_{\delta}}{c_{\delta}}\right)+t \frac{d c_{\delta}}{c_{\delta}}
$$

such that $t \frac{d c_{\delta}}{c_{\delta}}+d(v):=w^{\prime} \in \Omega_{M,<\delta}^{n}$.

(i) Suppose that $c_{\delta(n)}=\sqrt{\alpha_{1}}$ : In this case, we have $w_{\delta}=0$, and thus $\wp\left(u_{\delta} \frac{d c_{\delta}}{c_{\delta}}\right) \in$ $d \Omega_{M}^{n-1}+\Omega_{M,<\delta}^{n}$. By Kato's lemma, $u_{\delta} \frac{d c_{\delta}}{c_{\delta}}=\frac{d e_{\delta(1)}}{e_{\delta(1)}} \wedge \cdots \wedge \frac{d e_{\delta(n)}}{e_{\delta(n)}}+u^{\prime}$, where $e_{\delta(j)} \in$ $M_{\delta(j)}$ and $u^{\prime} \in \Omega_{M,<\delta}^{n}$. By substituting the expression $u_{\delta} \frac{d c_{\delta}}{c_{\delta}}$ in (2) and using the fact that $\wp\left(\frac{d e_{\delta(1)}}{e_{\delta(1)}} \wedge \cdots \wedge \frac{d e_{\delta(n)}}{e_{\delta(n)}}\right) \in d \Omega_{M}^{n-1}$, we are reduced to the case where $c_{\delta(n)} \in F$.

(ii) Suppose that $c_{\delta(n)} \in F$ : In this case, we have $v \in \Omega_{F}^{n-1}$. Otherwise, $v=v_{0}+$ $\sqrt{\alpha_{1}} v_{1}$ with $v_{0}, v_{1} \in \Omega_{F}^{n-1}$ and $v_{1} \neq 0$. Then, $d(v)=d\left(v_{0}\right)+\sqrt{\alpha_{1}} d\left(v_{1}\right)+v_{1} \wedge d\left(\sqrt{\alpha_{1}}\right)$. Consequently, $\frac{d c_{\delta}}{c_{\delta}}$ contains the slot $d\left(\sqrt{\alpha_{1}}\right)$, which is not possible. Hence, $t \frac{d c_{\delta}}{c_{\delta}}$ and $w^{\prime}$ belong to $\Omega_{F}^{n}$. In particular, $u_{\delta} \frac{d c_{\delta}}{c_{\delta}} \in \Omega_{F}^{n}$. Now substituting $w_{\delta} \frac{d c_{\delta}}{c_{\delta}}$ in $w$, we get

$$
\begin{aligned}
w & =w_{\delta} \frac{d c_{\delta}}{c_{\delta}}+w^{\prime \prime}, \quad \text { with } w^{\prime \prime} \in \Omega_{F,<\delta}^{n} \\
& =\wp\left(u_{\delta} \frac{d c_{\delta}}{c_{\delta}}\right)+d(v)+w^{\prime}+w^{\prime \prime} \\
& \equiv w^{\prime \prime \prime} \quad \bmod \wp\left(\Omega_{F}^{n}\right)+d \Omega_{F}^{n-1},
\end{aligned}
$$

with $w^{\prime \prime \prime}=w^{\prime}+w^{\prime \prime} \in \Omega_{F,<\delta}^{n}$. By iterating the procedure whenever $\delta(1)>m$, we are reduced to an element $w^{\prime \prime \prime}$ whose maximal multi-index $\lambda$ satisfies $\lambda(1) \leq m$. This means that $w^{\prime \prime \prime} \in \sum_{i=2}^{m} \frac{d \alpha_{i}}{\alpha_{i}} \wedge \Omega_{F}^{n-1}$. Hence, there exist $\tau_{2}, \cdots, \tau_{m} \in \Omega_{F}^{n-1}$, $r \in \Omega_{F}^{n-1}$ and $s \in \Omega_{F}^{n}$ such that, over the field $M$, we have

$$
w=\sum_{i=2}^{m} \frac{d \alpha_{i}}{\alpha_{i}} \wedge \tau_{i}+\wp(r)+d(s) .
$$

Since $\operatorname{Ker}\left(H_{2}^{n+1}(F) \longrightarrow H_{2}^{n+1}(M)\right)=\overline{\frac{d \alpha_{1}}{\alpha_{1}} \wedge \Omega_{F}^{n-1}}$, there exists $\tau_{1} \in \Omega_{F}^{n-1}$ such that $\bar{w}=\overline{\sum_{i=1}^{m} \frac{d \alpha_{i}}{\alpha_{i}} \wedge \tau_{i}}$.

The proof of Theorem 2 will be based on the following proposition:

\footnotetext{
${ }^{1}$ A mistake in the proof of [L2 Prop. 1] has been made when trying to make this descent. More precisely, it happens in [L2, page 2484, lines 7-8] when we tried to apply the Artin-Schreier operator.
} 
Proposition 3. Let $\alpha_{1}, \cdots, \alpha_{m}, a \in F$, and let $L=F\left(\sqrt{\alpha_{1}}, \cdots, \sqrt{\alpha_{m}}\right), M=$ $F\left(\wp^{-1}(a)\right)$. Then we have the following:

$$
\operatorname{Ker}\left(H_{2}^{n+1}(F) \longrightarrow H_{2}^{n+1}(L \cdot M)\right)=a \nu_{F}(n)+\sum_{i=1}^{m} \overline{d \alpha_{i} \wedge \Omega_{F}^{n-1}} .
$$

Proof. Without loss of generality, we may suppose that $[L: F]=2^{m}, a \in F \backslash \wp(F)$ and $a \in F^{2}$. Put $\beta=\wp^{-1}(a)$. Let $\mathcal{B}:=\left\{c_{1}, \cdots, c_{m}, c_{m+1}, \cdots\right\}$ be a 2 -basis of $F$ such that $c_{i}=\alpha_{i}$ for $i=1, \cdots, m$. Since $M / F$ is separable, $\mathcal{B}$ remains a 2 -basis of $M$. Then $\Omega_{M,<\delta}^{n}=\Omega_{F,<\delta}^{n}+\beta \Omega_{F,<\delta}^{n}$.

Let $\omega \in \Omega_{F}^{n}$ be such that $\bar{w} \in \operatorname{Ker}\left(H_{2}^{n+1}(F) \longrightarrow H_{2}^{n+1}(L \cdot M)\right)$. Then, over $M$, $\bar{\omega} \in \operatorname{Ker}\left(H_{2}^{n+1}(M) \longrightarrow H_{2}^{n+1}(L \cdot M)\right)$. It follows from Proposition 2 that

$$
\omega=\sum_{i=1}^{m} \sigma_{i} \wedge d \alpha_{i}+\wp(u)+d(v)
$$

with $u \in \Omega_{M}^{n}, v \in \Omega_{M}^{n-1}$, and $\sigma_{1}, \cdots, \sigma_{m} \in \Omega_{M}^{n-1}$. Let $\delta$ be the maximal multi-index in relation (3).

Suppose that $\delta(1)>m$. Then, as in the relation (2), there exists $w^{\prime} \in \Omega_{M,<\delta}^{n}$ such that

$$
w_{\delta} \frac{d c_{\delta}}{c_{\delta}}=\wp\left(u_{\delta} \frac{d c_{\delta}}{c_{\delta}}\right)+d(v)+w^{\prime} .
$$

Let $r_{\delta}, s_{\delta} \in F, v_{0}, v_{1} \in \Omega_{F}^{n-1}$, and $w_{0}^{\prime}, w_{1}^{\prime} \in \Omega_{F,<\delta}^{n}$ be such that $u_{\delta}=r_{\delta}+\beta s_{\delta}$, $v=v_{0}+\beta v_{1}$ and $w^{\prime}=w_{0}^{\prime}+\beta w_{1}^{\prime}$. A simple computation shows that the relation (41) gives the following:

$$
\left\{\begin{array}{l}
w_{\delta} \frac{d c_{\delta}}{c_{\delta}}=\wp\left(r_{\delta} \frac{d c_{\delta}}{c_{\delta}}\right)+d\left(v_{0}\right)+a s_{\delta}^{2} \frac{d c_{\delta}}{c_{\delta}}+w_{0}^{\prime}, \\
\wp\left(s_{\delta} \frac{d c_{\delta}}{c_{\delta}}\right)=d\left(v_{1}\right)+w_{1}^{\prime} .
\end{array}\right.
$$

By applying Kato's lemma to the second relation in (5), we get

$$
s_{\delta} \frac{d c_{\delta}}{c_{\delta}}=\frac{d a_{\delta(1)}}{a_{\delta(1)}} \wedge \cdots \wedge \frac{d a_{\delta(n)}}{a_{\delta(n)}}+w_{2}^{\prime},
$$

for some $w_{2}^{\prime} \in \Omega_{F,<\delta}^{n}$ and $a_{\delta(j)} \in F_{\delta(j)}$. Again with the second relation in (5), we obtain

$$
\begin{aligned}
a s_{\delta}^{2} \frac{d c_{\delta}}{c_{\delta}} & =a s_{\delta} \frac{d c_{\delta}}{c_{\delta}}+a d\left(v_{1}\right)+a w_{1}^{\prime} \\
& =a \frac{d a_{\delta(1)}}{a_{\delta(1)}} \wedge \cdots \wedge \frac{d a_{\delta(n)}}{a_{\delta(n)}}+d\left(a v_{1}\right)+a w_{1}^{\prime}+a w_{2}^{\prime} .
\end{aligned}
$$

If we substitute the expression $a s_{\delta}^{2} \frac{d c_{\delta}}{c_{\delta}}$ in the first relation in (5), we get

$$
w_{\delta} \frac{d c_{\delta}}{c_{\delta}}=\wp\left(r_{\delta} \frac{d c_{\delta}}{c_{\delta}}\right)+d\left(v^{\prime}\right)+a \eta_{\delta}+w^{\prime \prime},
$$

where $v^{\prime}=v_{0}+a v_{1}, \eta_{\delta}=\frac{d a_{\delta(1)}}{a_{\delta(1)}} \wedge \cdots \wedge \frac{d a_{\delta(n)}}{a_{\delta(n)}}$, and $w^{\prime \prime}=a w_{1}^{\prime}+a w_{2}^{\prime}+w_{0}^{\prime} \in \Omega_{F,<\delta}^{n}$.

Since $a \eta_{\delta} \in \wp\left(\Omega_{M}^{n}\right) \subset \wp\left(\Omega_{M}^{n}\right)+d\left(\Omega_{M}^{n-1}\right)$, the multi-index has been reduced. By iterating the procedure whenever $\delta(1)>m$, we obtain, over $M$, the following:

$$
w=\wp\left(\gamma_{1}\right)+d\left(\gamma_{2}\right)+a \gamma_{3}+\gamma_{4},
$$

for $\gamma_{1} \in \Omega_{F}^{n}, \gamma_{2} \in \Omega_{F}^{n-1}, \gamma_{3} \in \nu_{F}(n)$ and $\gamma_{4} \in \Omega_{F}^{n}$, with the condition that the maximal multi-index $\lambda$ in $\gamma_{4}$ satisfies $\lambda(1) \leq m$. Then $\gamma_{4} \in \sum_{i=1}^{m} d \alpha_{i} \wedge \Omega_{F}^{n-1}$. Now, 
since the kernel $\operatorname{Ker}\left(H_{2}^{n+1}(F) \longrightarrow H_{2}^{n+1}(M)\right)=\overline{a \nu_{F}(n)}$ [AB, we get the desired conclusion.

As a corollary, we get:

Corollary 1. Let $n \geq 1$ be an integer, and let $\alpha_{1}, \cdots, \alpha_{m}, a \in F$. Let $L=$ $F\left(\sqrt{\alpha_{1}}, \ldots, \sqrt{\alpha_{m}}\right)$ and $M=L\left(\wp^{-1}(a)\right)$. Then we have:

(1) $\operatorname{Ker}\left(\overline{I^{n}} W_{q}(F) \longrightarrow \overline{I^{n}} W_{q}(L)\right)=\sum_{i=1}^{m} \overline{\left\langle 1, \alpha_{i}\right\rangle_{b} \otimes I^{n-1} W_{q}(F)}$.

(2) $\operatorname{Ker}\left(\overline{I^{n}} W_{q}(F) \longrightarrow \overline{I^{n}} W_{q}(L \cdot M)\right)=\overline{I^{n} F \otimes[1, a]}+\sum_{i=1}^{m} \overline{\left\langle 1, \alpha_{i}\right\rangle_{b} \otimes I^{n-1} W_{q}(F)}$.

Proof. We use Propositions 2 and 3 with the commutativity of the diagram

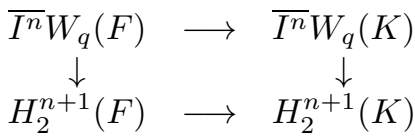

where $K=L($ resp. $K=L \cdot M)$, the vertical arrows are given by the isomorphism $e^{n}$, and the horizontal arrows are induced by the inclusion $F \subset K$.

Proof of Theorems 1] and 2. We use Corollary 1] and we follow the same argument used for the proof of [L2, Theorem 1].

We also have the following corollary, whose proof is the same as for Theorems 1 and 2 .

Corollary 2. Let $n \geq 1$ be an integer, and let $\alpha_{1}, \cdots, \alpha_{m}, a \in F$. Let $L=$ $F\left(\sqrt{\alpha_{1}}, \ldots, \sqrt{\alpha_{m}}\right)$ and $M=L\left(\wp^{-1}(a)\right)$. Then we have:

(1) $\operatorname{Ker}\left(I^{n} W_{q}(F) \longrightarrow I^{n} W_{q}(L)\right)=\sum_{i=1}^{m}\left\langle 1, \alpha_{i}\right\rangle_{b} \otimes I^{n-1} W_{q}(F)$.

(2) $\operatorname{Ker}\left(I^{n} W_{q}(F) \longrightarrow I^{n} W_{q}(L \cdot M)\right)=I^{n} F \otimes[1, a]+\sum_{i=1}^{m}\left\langle 1, \alpha_{i}\right\rangle_{b} \otimes I^{n-1} W_{q}(F)$.

\section{ACKNOWLEDGEMENT}

The work for this note was done while the first author was a visiting researcher at Université d'Artois, Lens, France, which he would like to thank for its hospitality.

\section{REFERENCES}

[Ah] Hamza Ahmad, Witt kernels of bi-quadratic extensions in characteristic 2, Bull. Austral. Math. Soc. 69 (2004), no. 3, 433-440, DOI 10.1017/S0004972700036212. MR2066661 (2005g:11058a)

[AB] R. Aravire and R. Baeza, The behavior of quadratic and differential forms under function field extensions in characteristic two, J. Algebra 259 (2003), no. 2, 361-414, DOI 10.1016/S0021-8693(02)00568-9. MR1955526(2003k:11060)

[B] Ricardo Baeza, Quadratic forms over semilocal rings, Lecture Notes in Mathematics, Vol. 655, Springer-Verlag, Berlin, 1978. MR0491773 (58 \#10972)

$[\mathrm{H}] \quad$ Detlev W. Hoffmann, Witt kernels of bilinear forms for algebraic extensions in characteristic 2, Proc. Amer. Math. Soc. 134 (2006), no. 3, 645-652 (electronic), DOI 10.1090/S00029939-05-08175-X. MR2180880 (2006h:11045)

[DH] A. Dolphin and D. W. Hoffmann, Differential forms and bilinear forms under field extensions, preprint, 2011.

[K] Kazuya Kato, Symmetric bilinear forms, quadratic forms and Milnor K-theory in characteristic two, Invent. Math. 66 (1982), no. 3, 493-510, DOI 10.1007/BF01389226. MR662605 (83i:10027) 
[L1] Ahmed Laghribi, Witt kernels of function field extensions in characteristic 2, J. Pure Appl. Algebra 199 (2005), no. 1-3, 167-182, DOI 10.1016/j.jpaa.2004.12.005. MR2134299 (2006c:11041)

[L2] Ahmed Laghribi, Witt kernels of quadratic forms for purely inseparable multiquadratic extensions in characteristic 2, Proc. Amer. Math. Soc. 134 (2006), no. 9, 2481-2486 (electronic), DOI 10.1090/S0002-9939-06-08649-7. MR2213724(2007d:11040)

[MM] Pasquale Mammone and Remo Moresi, Formes quadratiques, algèbres à division et extensions multiquadratiques inséparables, Bull. Belg. Math. Soc. Simon Stevin 2 (1995), no. 3, 311-319 (French, with French summary). MR:1338463|(96j:11052)

Universidad Arturo Prat, Casilla 121, Iquique, Chile

E-mail address: raravire@unap.cl

Université d'Artois, Faculté des Sciences Jean Perrin, Laboratoire de mathématiques De Lens - EA2462, rue Jean Souvraz - SP18, F-62307 Lens, France

E-mail address: laghribi@euler.univ-artois.fr 